\title{
La insatisfacción con la democracia en América Latina Análisis de factores económicos y políticos en 2017
}

\author{
Dissatisfaction with democracy in Latin America. \\ Analysis of economic and political factors in 2017
}

Yissel Santos González

Universidad Iberoamericana

yisselsantos87@gmail.com

Código Orcid: https://orcid.org/0000-0001-6612-5621

Oscar A. Martínez-Martínez

Universidad Iberoamericana

oscar.martinez@ibero.mx

Código Orcid: https://orcid.org/0000-0003-4103-674X

\begin{abstract}
Resumen
Los bajos índices de satisfacción con el funcionamiento de la democracia en Latinoamérica desde inicios de siglo, ha motivado a investigar las causas que originan estos resultados. Por esa razón, el objetivo del artículo fue, explicar qué factores inciden en la percepción de satisfacción con el funcionamiento de la democracia en dicha región. Para comprobarlo, se aplicó un modelo de regresión logístico binaria, a ocho variables agrupadas en tres dimensiones: rendimiento económico, político y percepción de corrupción; la base de datos utilizada fue el Latinobarómetro 2017. Los resultados muestran que tanto los factores económicos como los políticos, generan mayor probabilidad de estar insatisfechos con el funcionamiento de la democracia, a pesar de esto, las personas siguen considerando a la democracia como la mejor forma de gobierno.
\end{abstract}

\section{Palabras clave}

Satisfacción con la democracia, instituciones políticas, economía, corrupción, Latinoamérica.

Forma sugerida de citar: Santos González, Y., y Martínez-Martínez, Oscar A., (2020). La insatisfacción con la democracia en América Latina. Análisis de factores económicos y políticos en 2017. Universitas, 32, pp. 157-174. 


\begin{abstract}
The low satisfaction rates with the functioning of democracy in Latin America since the beginning of the century, has motivated to investigate the causes that originate these results. For that reason, the objective of the article is to explain what factors affect the perception of satisfaction with the functioning of democracy in that region. To verify this, a binary logistic regression model was applied to eight variables grouped into three dimensions: economic, political performance and perception of corruption; The database used was the Latinobarometer 2017. The results show that both economic and political factors generate a greater probability of being dissatisfied with the functioning of democracy, despite this, people continue to consider democracy as the best form of government.
\end{abstract}

Keywords

Satisfaction with democracy, political institutions, economy, corruption, Latin America.

\title{
Introducción
}

La comprensión teórica de la democracia ha acompañado a lo largo de los siglos la labor de disímiles pensadores desde distintas posiciones epistémicas e ideológicas. Durante la segunda mitad del siglo XX, Latinoamérica vivió procesos que consolidaron la democracia como el sistema de gobierno sucesor a regímenes autoritarios en varios países del continente, específicamente en el Cono Sur, pero esto no quiere decir que se constituyeran como democracias perfectas. Las democracias que nacen en América Latina durante la década de los ochenta, han tenido como una de sus tareas políticas, construir y consolidar el neoliberalismo (Dávalos, 2008), proceso que condicionó las democracias de toda la región y hoy sigue haciéndolo.

La democracia se ha desarrollado por lo regular en dos niveles, el político, como forma de organización de la sociedad que incluye la toma de decisiones para el bien de las mayorías, y como forma de vida, convirtiéndose en una comunidad social de carácter ético, y sociocultural que determina la acción de los ciudadanos (Dunn, 2014). Cuando estos factores no operan correctamente estamos ante una crisis o ausencia de condiciones democráticas 
que a su vez reducen la satisfacción de la ciudadanía con el gobierno; esto puede explicar en cierta medida, por qué ha disminuido la satisfacción con la democracia en Latinoamérica, pues entre 2016 y 2017, decreció del 34\% a 30\% (Latinobarómetro, 2017), esto pudiera señalar un malestar con la democracia y las instituciones (Galli, 2013).

En la lógica anterior, lleva a cuestionar, la confianza en instituciones políticas y de gobierno, la distribución del ingreso, el grado de libertades y derechos ciudadanos, el desempeño del sistema electoral, los cuales marcan una pauta para la satisfacción o insatisfacción democrática (Díaz, 2014). Por esa razón, el objetivo de este artículo es analizar cómo los factores de rendimiento económico, unido a los factores de rendimiento político y la percepción de corrupción, explican la satisfacción o insatisfacción con el funcionamiento de la democracia en Latinoamérica.

El artículo se encuentra dividido en cuatro apartados, primero, revisión de la literatura, que exponen desde diferentes teorías, los principales elementos que explican satisfacción con el funcionamiento de la democracia; segundo, metodología, donde se describen: las variables y base de datos utilizada en el estudio, así como la técnica ocupada; tercero, resultados, donde se exponen los hallazgos del modelo estadístico; cuarto, discusión, donde analizamos los resultados en relación a la literatura; finalmente, las conclusiones.

\section{Revisión de la literatura}

El constante descenso de la calidad y funcionamiento de la democracia en América Latina, ha sido un tema de recurrente análisis en la última década. En cambio, a pesar que la satisfacción con la democracia, ha sido uno de los conceptos más usados para el análisis político, no existe consenso respecto a las dimensiones que involucran este concepto (Canache, Mondak \& Sligson, 2001). De acuerdo al Programa de las Naciones Unidas para el Desarrollo (2004) la satisfacción de la democracia ha resultado muy controversial a la hora de explicar qué factores la determinan; debido a que es una variable de desempeño y mide tanto la evaluación del régimen político como la evaluación del gobierno o del partido que esté en el poder, más aún como lo señala PNUD, en Latinoamérica, se presenta un malestar y desencanto no necesariamente con la democracia, pero sí dentro de ella, debido a que los ciudadanos distinguen entre democracia como sistema de gobierno y el desempeño de los gobernantes en particular. 
El presupuesto anterior es comprensible si se entiende a la democracia moderna como aquella en la que todos los ciudadanos comparten un conjunto de condiciones iguales ante la ley, que les permita ejercer la construcción de la misma, al conceder poder a determinada figura política, así como a la política misma (Dunn, 2014). En cambio el problema está en el ejercicio de sus derechos ciudadanos ante la ley y la ciudadanía universal (Galli, 2013). Esto es lo que llama Estado representativo de derecho, como parte integrante de la democracia moderna, como señala este último autor.

Se trata de una transferencia de poder político, donde el ciudadano, ejerce su soberanía al delegar su voluntad en unos pocos que lo representarán, y solo así se reconoce como universal, pero, cuando el ciudadano no siente correspondencia entre ese poder que ha otorgado a determinado representante político o figura gubernativa, experimenta malestar o insatisfacción hacia el funcionamiento político que creía lo representaba (Galli, 2013), lo cual produce, la insatisfacción política hacia el funcionamiento del sistema democrático que lo representa. El malestar con la democracia se desarrolla en dos niveles. El primero se muestra a través de la apatía política del sujeto, mediante el desencanto o desconfianza hacia quienes lo representan, así como a la democracia como representación de sus intereses. El segundo, es de carácter objetivo, el cual va vinculado a "la inadecuación de la democracia de mantener sus instituciones, en la incapacidad de cumplir sus promesas y de regular la política" (Galli, 2013, p. 10). Llevando a que el ciudadano se sienta insatisfecho y sin alternativas políticas.

Autores como (Díaz, 2014; Barreda, 2011) han intentado explicar qué elementos determinan en la satisfacción con el funcionamiento de la democracia. Estos coinciden en dos elementos: el rendimiento económico y el rendimiento del sistema político, donde incluyen, el funcionamiento de instituciones políticas, la confianza en ellas, la valoración sobre las personas si están de acuerdo o no con la democracia como sistema de gobierno, o cómo consideran que es ésta en su país. Además de las dimensiones relativas a los procedimientos democráticos y al control del poder político, también se incluyen indicadores, como pueden ser la igualdad socioeconómica o justa distribución del ingreso, el nivel de bienestar o la justicia social.

En ese sentido, la perspectiva del rendimiento económico sostiene que los diferentes niveles de satisfacción con el funcionamiento de la democracia están asociados a los resultados de la economía, y pueden responder tan- 
to a indicadores medidos a nivel nacional (crecimiento económico) como personal (mejora de sus condiciones o recursos económicos), y derivado de ello, también responderían a la valoración de la efectividad del gobierno en la gestión de los asuntos económicos (Díaz, 2014). A mayor crecimiento económico de un país, la probabilidad de consolidar un régimen democrático es mayor y aumenta el grado de satisfacción de las personas (Lipset, 1959; Scott Mainwaring \& Pérez-Liñán, 2008; Barreda, 2011).

Bajo esa misma lógica, los altos niveles de desigualdad social como los que presenta América Latina, unido a la no equidad distributiva del ingreso per cápita, disminuyen la satisfacción democrática, a la vez que el apoyo expresado hacia la autoridad democrática en América Latina (Carlin, 2006). Al respecto, Schumpeter (1942) y Sen (1999), señalan que el orden democrático va ligado al crecimiento de la economía, constituyendo la estabilidad de las instituciones y capacidad de avance social, ambos autores sientan la base de una tradición teórica que ha propuesto largamente la importancia de la situación económica del país como el factor más influyente en la satisfacción con la democracia.

Situado en este eje analítico, Zovatto (2018) incluye el elemento de la desigualdad como factor de quiebre de una democracia, a pesar que reconoce que, desde hace 39 años, América Latina vive el proceso de democratización o (re)democratización (según el país en cuestión) más largo, extenso y profundo de toda su historia. Pero ello, señala:

(...) la democracia en América Latina presenta una paradoja: es la única región del mundo donde existe una combinación de regímenes democráticos en la casi totalidad de los países que la integran, con amplios sectores de su población viviendo por debajo de la línea de la pobreza (cerca del 30\% para 2017, según la CEPAL, 2016), la distribución del ingreso más desigual del mundo, las tasas de homicidios más elevadas del planeta y muy altos niveles de corrupción. (Zovatto, 2018, p. 4)

En cuanto al rendimiento político como un segundo factor que explica la satisfacción con la democracia, Aarts y Thomasen (2008) señalan que la variabilidad en el grado de satisfacción con el funcionamiento de la democracia tiene una cadena causal que se inicia en elementos institucionales. Por su parte Anderson y Guillory (1997) destacan la relevancia de las preferencias políticas de los ciudadanos y su aprobación al presidente en turno, 
dado el grado de presidencialismo en América Latina; en la medida que se sientan representados por ese partido o presidente al que han elegido para ejercer el poder en nombre de todos, se sentirán más satisfechos con el funcionamiento de la democracia como sistema de gobierno. En esa misma línea, O’Donnell señala:

(...) la democracia no es tan sólo un régimen democrático, sino también un modo particular de relación, entre Estado y ciudadanos y entre los propios ciudadanos, bajo un tipo de estado de derecho que, junto con la ciudadanía política, sostiene la ciudadanía civil y una red completa de rendición de cuentas. (O’Donnell, 2001, p. 27)

La teoría institucionalista señala que la causa de quiebre de la democracia y la poca satisfacción se debe a la fragilidad de las instituciones así como a un diseño inadecuado (Espino, 2002). Es por ello, que un indicador que permite medirlo es la confianza en las instituciones, por esa razón, cuando los ciudadanos expresan altos niveles de confianza en el parlamento, tribunales, gobiernos, municipios, policías y partidos políticos, probablemente existirá una mayor satisfacción con el sistema democrático (Cea, Alister \& Guerrero, 2015).

Otro de los indicadores que puede asociarse con la satisfacción con la democracia es la percepción de corrupción, al respecto Cea, Alister y Guerrero (2015) señalan que existe amplio consenso en que la corrupción genera un impacto negativo en el apoyo a los sistemas políticos. Destacan la importancia de integrar la noción de corrupción al análisis de la satisfacción con la democracia pues los sujetos que:

Tienen alta percepción de corrupción, (...) se muestran abiertamente insatisfechos con su funcionamiento, por lo que los niveles de corrupción no sólo dañan al gobierno de turno, sino que también deprimen los índices de satisfacción con la democracia. (Cea, Alister \& Guerrero, 2015, p. 93)

De igual forma, Morales (2009) expone que la percepción de la corrupción responde a esas ideas o imágenes que se crean respecto al desempeño de las autoridades y, que en América Latina posee una específica relevancia, pues el presidencialismo caracteriza las democracias de la región, esto puede reflejar un malestar que manifiesta la falta de satisfacción con la democracia frente a la valoración del presidente en el país (Cereceda-Maram- 
bio \& Torres-Solís, 2017). La corrupción y escándalos políticos, deterioran la calidad del sistema político, a la vez, afectan la satisfacción democrática, erosionando paulatinamente sus niveles de apoyo ciudadano y afectan la imagen de los partidos políticos implicados (Sotillo, 2015). Cuestión que adquiere especial relevancia en América Latina en la última década y se ve expresado en ese malestar, como señala Zovatto:

(...) ha producido un aumento de insatisfacción ciudadana con el funcionamiento de la democracia y hacia las élites, expresada en algunos países mediante un amplio descontento popular (el llamado "efecto calle"), lo que ha generado crisis de gobernabilidad de diferente intensidad y consecuencias, llegando incluso, en algunos países, a ocasionar la finalización anticipada de los mandatos constitucionales de más de 15 presidentes entre 1978 y 2016. (Zovatto, 2018, p. 5)

El funcionamiento de las democracias en América Latina no escapa del funcionamiento en general del sistema que debe ser comprendido en su relación hegemónica y polarizadora. Aspectos que explican su quiebre y bajos índices de satisfacción ciudadana, al constituir la región más desigual del mundo (Zovatto, 2011). Por esa razón, es necesario analizar la satisfacción/ insatisfacción de la democracia, tomando en cuenta las categorías económicas, políticas y la corrupción.

\section{Materiales y métodos}

El estudio utilizó el Latinobarómetro (2017), que tiene una muestra representativa, con 20200 observaciones, la cual está distribuida en 18 países: Argentina, Bolivia, Chile, Colombia, Ecuador, México, Brasil, Paraguay, Perú y Uruguay, se aplicaron en cada país, 1200 cuestionarios; en Costa Rica, El Salvador, Guatemala, Honduras, Nicaragua, Panamá y República Dominicana.

A partir de nuestro objetivo "analizar cómo los factores de rendimiento económico, unido a los factores de rendimiento político y la percepción de corrupción, explican la satisfacción o insatisfacción con el funcionamiento de la democracia en Latinoamérica" las tres dimensiones: rendimiento económico, rendimiento político y percepción de corrupción; la variable dependiente fue la satisfacción con la democracia. 


\section{Cuadro 1 \\ Variables del modelo estadístico}

\begin{tabular}{|c|c|c|c|}
\hline Dimensiones & $\begin{array}{c}\text { Tipo de } \\
\text { variables }\end{array}$ & Variables & Valores de respuesta \\
\hline $\begin{array}{l}\text { Satisfacción con el } \\
\text { funcionamiento de la } \\
\text { democracia }\end{array}$ & $\begin{array}{c}\text { Dependiente } \\
Y Y\end{array}$ & $\begin{array}{l}\text { Satisfacción con el } \\
\text { funcionamiento de la } \\
\text { democracia (binomial) }\end{array}$ & $\begin{array}{l}\text { 0. No satisfecho con el } \\
\text { funcionamiento de la democracia } \\
\text { 1. Satisfecho con el funcionamiento } \\
\text { de la democracia }\end{array}$ \\
\hline \multirow{2}{*}{$\begin{array}{l}\text { Rendimiento } \\
\text { económico }\end{array}$} & $\begin{array}{l}\text { Independiente } \\
\qquad \mathrm{X}_{1}\end{array}$ & $\begin{array}{l}\text { Situación económica } \\
\text { actual del país } 3 \text { (Ordinal) }\end{array}$ & $\begin{array}{l}\text { 1. Muy mala } \\
\text { 2. Mala } \\
\text { 3. Regular } \\
\text { 4. Buena } \\
\text { 5. Muy buena }\end{array}$ \\
\hline & $\begin{array}{c}\text { Independiente } \\
\mathrm{X}_{2}\end{array}$ & $\begin{array}{l}\text { Funcionamiento de la } \\
\text { economía } 2\end{array}$ & $\begin{array}{l}\text { 1. Nada satisfecho } \\
\text { 2. No muy satisfecho } \\
\text { 3. Más bien satisfecho } \\
\text { 4. Muy satisfecho }\end{array}$ \\
\hline \multirow{4}{*}{ Rendimiento político } & $\begin{array}{l}\text { Independiente } \\
\qquad \mathrm{X}_{3}\end{array}$ & $\begin{array}{l}\text { Grado de confianza en el } \\
\text { gobierno } 2\end{array}$ & $\begin{array}{l}\text { 1. Ninguna } \\
\text { 2. Poca } \\
\text { 3. Algo } \\
\text { 4. Mucha }\end{array}$ \\
\hline & $\begin{array}{l}\text { Independiente } \\
\mathrm{X}_{4}\end{array}$ & $\begin{array}{l}\text { ¿Cómo de justa es la } \\
\text { distribución del ingreso } \\
\text { en tu país? } 2\end{array}$ & $\begin{array}{l}\text { 1. Muy injusta } \\
\text { 2. Injusta } \\
\text { 3. Justa } \\
\text { 4. Muy justa }\end{array}$ \\
\hline & $\begin{array}{l}\text { Independiente } \\
\mathrm{X}_{5}\end{array}$ & $\begin{array}{l}\text { Grado de acuerdo. } \\
\text { La democracia puede } \\
\text { tener problemas, pero } \\
\text { es el mejor sistema de } \\
\text { gobierno } 2\end{array}$ & $\begin{array}{l}\text { 1. Muy en desacuerdo } \\
\text { 2. En desacuerdo } \\
\text { 3. De acuerdo } \\
\text { 4. Muy de acuerdo }\end{array}$ \\
\hline & $\begin{array}{l}\text { Independiente } \\
\mathrm{X}_{6}\end{array}$ & $\begin{array}{l}\text { Aprobación de la } \\
\text { gestión del gobierno que } \\
\text { encabeza el presidente } 2\end{array}$ & $\begin{array}{l}\text { 1. No aprueba } \\
\text { 2. Aprueba }\end{array}$ \\
\hline \multirow{2}{*}{$\begin{array}{l}\text { Percepción de } \\
\text { corrupción }\end{array}$} & $\begin{array}{l}\text { Independiente } \\
\mathrm{X}_{7}\end{array}$ & $\begin{array}{l}\text { ¿Cuánto cree Ud. que se } \\
\text { ha progresado en reducir } \\
\text { la corrupción en las } \\
\text { instituciones del estado } \\
\text { en estos últimos } 2 \text { años? }\end{array}$ & $\begin{array}{l}\text { 1. Nada } \\
\text { 2. Algo } \\
\text { 3. Poco } \\
\text { 4. Mucho }\end{array}$ \\
\hline & $\begin{array}{l}\text { Independiente } \\
\mathrm{X}_{8}\end{array}$ & $\begin{array}{l}\text { País gobernado por } \\
\text { unos cuantos grupos } \\
\text { poderosos en su propio } \\
\text { beneficio }\end{array}$ & $\begin{array}{l}\text { 1. Grupos poderosos en su propio } \\
\text { beneficio } \\
\text { 2. Para el bien de todo el pueblo }\end{array}$ \\
\hline
\end{tabular}

Fuente: Elaboración propia con datos del Latinobarómetro, 2017. 
La técnica utilizada fue una regresión logística binaria, debido a que la variable dependiente es de tipo dicotómica. El ajuste del modelo resultó significativo, tanto en la Chi Cuadrada ( $\mathrm{p}<0.005)$, la prueba ómnibus de coeficientes de modelo $(\mathrm{p}<0.000)$ y la prueba Hosmer y Lameshow $(\mathrm{p}<0.000)$

\section{Resultados}

En el cuadro 2, están los resultados del modelo logístico, como se observa los $\operatorname{Exp}(B)$ están en un intervalo de confianza del 95\%, mayores a uno, todos con signo positivo, y significativos $(\mathrm{p}<.00)$. Los resultados están ordenados de mayor a menor peso, en cuanto a la probabilidad de sentirse satisfecho (1) o no satisfecho (0) con el funcionamiento de la democracia en Latinoamérica. Las variables con mayor peso son las que integran la dimensión del rendimiento económico. Por una parte, el funcionamiento de la economía, es la más relevante, aumenta en 2511 veces la probabilidad de estar satisfecho con el funcionamiento de la democracia. En ese mismo sentido, la percepción de mejor situación económica actual del país aumenta en 1258 veces la probabilidad de estar satisfecho.

En cuanto a la distribución del ingreso, solo el $19.3 \%$ la considera justa, de ahí que nuestros resultados indiquen que al aumentar la percepción sobre la justa distribución del ingreso en un país, aumenta en 1326 veces la probabilidad de que la población esté satisfecha con el funcionamiento de la democracia, asociando a esta última como una forma de gobierno que impulsa la equidad o la distribución igualitaria de la riqueza. Lo anterior podría explicar, porque solo el $12.4 \%$ de la población considera que es buena la situación económica y un $1.2 \%$ que es muy buena, en ese sentido este indicador puede asociarse con los niveles de pobreza, desigualdad y al mismo tiempo de insatisfacción con el funcionamiento de la democracia y con la política en general.

Por otra parte, en el informe de Latinobarómetro (2017) es constatable que la percepción que se gobierna para los intereses de unos pocos, aumentó por segundo año consecutivo de $73 \%$ en 2016 a $75 \%$ en 2017. En el caso del modelo estadístico, esta variable fue la segunda con mayor peso, mostrando que la probabilidad de estar satisfecho con el funcionamiento de la democracia, aumenta en 1792 veces conforme un país es gobernado para el bien de todo el pueblo y no solamente para los grupos poderosos en su propio be- 
neficio. Se establece una relación directa que se confirma con la literatura, pues de acuerdo con Dunn (2014) la democracia es un valor social asociado a los intereses del pueblo, es por ello, que los gobiernos son crecientemente criticados porque no defienden los intereses de la mayoría, en cambio se sumen cada vez más, en fraudes políticos y en procesos de corrupción. Los hallazgos permiten señalar, además, que independientemente del descenso paulatino de la satisfacción con el funcionamiento de la democracia, el "Grado de acuerdo, la democracia puede tener problemas, pero es el mejor sistema de gobierno", indica que en la medida que se esté de acuerdo con la democracia como mejor sistema de gobierno, aumenta en 1639 veces la probabilidad de estar satisfecho con el funcionamiento de la democracia.

Lo anterior puede relacionarse, con la aprobación de la gestión del gobierno que encabeza el presidente, al respecto el 39.3\% señala que aprueba la gestión gubernamental de su presidente. Lo anterior permite explicar que la escasa confianza en el gobierno y la baja aprobación de la gestión del gobierno influyen en el descenso de la satisfacción con el funcionamiento de la democracia. Por esa razón, el valor de su Exp (B) señala que la aprobación de la gestión del gobierno que encabeza el presidente en turno en cada país aumenta en 1624 veces la probabilidad de la satisfacción con el funcionamiento de la democracia.

En esta misma línea, en Latinoamérica, el 33.3\% de la población tiene poca confianza en el gobierno y un $41.8 \%$ responde que ninguna confianza, lo cual es un dato muy revelador ante las fuertes demandas de la sociedad civil a esta institución, debido a la insatisfacción que ha venido presentándose en los últimos años, en ese sentido, nuestros resultados señalan que la confianza en el Gobierno, genera 1309 veces la probabilidad de estar satisfecho con el funcionamiento de la democracia, señalando la importancia que tiene esta variable para disminuir la insatisfacción con la democracia.

Por otra parte, teniendo en cuenta que uno de los problemas más importantes en Latinoamérica es la corrupción (Zovatto, 2018), los resultados señalan que al percibir que se está reduciendo la corrupción, aumenta en 1120 veces la probabilidad de estar satisfecho con el funcionamiento de la democracia. En ese sentido, la confianza en las instituciones políticas es un indicador de gran incidencia de acuerdo con la teoría (Ayala, 2002). 


\section{Cuadro 2}

\section{Resultados del modelo logístico}

\begin{tabular}{|l|l|l|l|l|l|l|}
\hline \multicolumn{1}{|c|}{ Variables de la ecuación } & B & $\begin{array}{c}\text { Error } \\
\text { estándar }\end{array}$ & Wald & gl & Sig. & Exp(B) \\
\hline Funcionamiento de la economía & .921 & .031 & 902.012 & 1 & .000 & 2.511 \\
\hline $\begin{array}{l}\text { País gobernado por unos cuantos grupos } \\
\text { poderosos en su propio beneficio }\end{array}$ & .583 & .052 & 123.808 & 1 & .000 & 1.792 \\
\hline $\begin{array}{l}\text { Grado de acuerdo. La democracia puede tener } \\
\text { problemas pero es el mejor sistema de gobierno }\end{array}$ & .494 & .031 & 253.943 & 1 & .000 & 1.639 \\
\hline $\begin{array}{l}\text { Aprobación de la gestión del gobierno que } \\
\text { encabeza el presidente }\end{array}$ & .485 & .048 & 100.163 & 1 & .000 & 1.624 \\
\hline $\begin{array}{l}\text { ¿Cómo de justa es la distribución del ingreso en } \\
\text { tu país? }\end{array}$ & .282 & .031 & 82.279 & 1 & .000 & 1.326 \\
\hline Grado de confianza en el gobierno & .269 & .027 & 100.154 & 1 & .000 & 1.309 \\
\hline Situación económica actual del país & .229 & .029 & 62.867 & 1 & .000 & 1.258 \\
\hline $\begin{array}{l}\text { ¿Cuánto cree Ud. que se ha progresado en reducir } \\
\text { la corrupción en las instituciones del estado en } \\
\text { estos últimos 2 años? }\end{array}$ & .113 & .023 & 24.940 & 1 & .000 & 1.120 \\
\hline Constante & 7.513 & .139 & 2933.43 & 1 & .000 & .001 \\
\hline
\end{tabular}

Fuente: Elaboración propia con datos del Latinobarómetro (2017)

\section{Discusión}

En Latinoamérica, la baja satisfacción con el funcionamiento de la democracia responde a factores tanto económicos como políticos, que se encuentran estrechamente vinculados en el ejercicio de gobierno. El mal funcionamiento de estos, ha generado desencanto respecto al funcionamiento de la democracia como sistema de gobierno, a pesar que el 69\% está de acuerdo en que no existe otra forma mejor, aun presentando problemas. A pesar de esto, el malestar y la insatisfacción están presentes, lo que deteriora la confianza de los ciudadanos en el gobierno y demás instituciones políticas y civiles.

Desde la teoría, Galli (2013) analiza este malestar hacia la democracia, fundamentalmente ante la imposibilidad del sistema democrático de mantener sus instituciones, regular la actividad política y cumplir sus promesas. Es importante aclarar, que no es un rechazo a la democracia como sistema 
de gobierno, sino, a su mal funcionamiento, que va unido a la crisis institucional del Estado en las últimas décadas. En este sentido, la crítica fundamental de la ciudadanía se vuelca hacia el tipo de gobierno que no representa sus intereses o incumple sus promesas, así como a la gestión del gobierno que encabeza el presidente en turno, pues estos niveles de percepción continúan disminuyendo. Lo anterior se ve explicado en el modelo, si tenemos en cuenta que estos indicadores, unidos al del mal funcionamiento de la economía, presentaron las betas más altas y por tanto los mayores índices en términos probabilísticos, por tanto, al incrementar la percepción negativa en esas variables incrementará la probabilidad de no estar satisfecho con el funcionamiento de la democracia en Latinoamérica.

Según el informe del Latinobarómetro (2017) la aprobación de los gobiernos ha descendido desde 2009, sumado a ello, el incremento de demandas sociales en todo el continente en las últimas décadas. Sus reclamos se centran en mayores cuotas de derechos, justicia social, libertades políticas de organización y expresión; que han permitido un auge de la acción colectiva, desde distintos espacios y dimensiones de la sociedad, con la finalidad de generar una real democratización, entendiéndose ésta, como la necesidad de desmercantilizar la sociedad, de cuestionar las formas dominantes del mercado y exigir los derechos esenciales como ciudadanos. Los resultados del modelo demuestran que en la medida que la población perciba que se gobierna de acuerdo con los intereses de todo el pueblo, aumentará su nivel de percepción a favor del funcionamiento de la democracia. En cambio, el indicador que refleja la percepción sobre si el país está gobernado por unos cuantos poderosos en su propio beneficio ha aumentado en los dos últimos años. El 75\% de los latinoamericanos perciben que se gobierna para unos cuantos grupos poderosos, aspecto que ha debilitado la confianza en el funcionamiento democrático.

En ese mismo orden de ideas Sotillo (2015) señala que una mejor percepción ciudadana de la función representativa de los partidos políticos, junto con un mayor sentimiento de eficacia gubernamental mejorará la valoración que se tiene de la democracia estrechando el vínculo representativo. Por el contrario, valoraciones más bajas en estas dos dimensiones tenderán a estabilizar sentimientos de insatisfacción democrática y, por ende, redundará en una falta de compromiso político entre los ciudadanos y peores valoraciones sobre el funcionamiento de la democracia. 
El fenómeno de la corrupción es uno de los problemas más graves en Latinoamérica, pues solo el $35.9 \%$ de la población tiene la percepción que se está progresando en reducirla, si tenemos en cuenta que es uno de los indicadores que mayor desconfianza genera en el funcionamiento democrático (Cereceda-Marambio \& Torres-Solís, 2017), por tanto, es necesario incluirlo como tema central en las agendas políticas y gubernamentales. Nuestros hallazgos muestran, que la disminución de la corrupción genera el aumento de la probabilidad de satisfacción con el funcionamiento a la democracia, como es el caso de Uruguay, que es el país con mayor satisfacción con la democracia y que presenta altos índices de progreso en combatir la corrupción, ya sea a través de políticas públicas o tomas de decisiones encaminadas a ello; el no tomar acciones para combatir la corrupción, erosionaría la satisfacción democrática y con ello el sistema de prácticas políticas de partidos y demás instituciones (Sotillo, 2015).

En cuanto a la percepción sobre la distribución del ingreso, solo el 19.3\% la considera justa, aspecto que influye en términos probabilísticos en la disminución de la satisfacción con el funcionamiento de la democracia. Elemento que se entiende si asumimos que 10 de los 15 países más desiguales del mundo se encuentran en América Latina (Zovatto, 2011), dándose lo que el autor clasifica como "trampa de desigualdad", pues el coeficiente de Gini del ingreso en la región es más elevado que el de países de África Subsahariana o algunos de Asia, en cambio registra en términos de desigualdad mayores desniveles. El alto nivel explicativo de esta variable puede relacionarse con la importancia que le conceden los latinoamericanos al factor de rendimiento económico. Es destacable que el funcionamiento de la economía resultó ser la de mayor nivel predictivo, lo que se corresponde con la postura de diversos autores (Díaz, 2014; Lipset, 1959). Unido a este indicador se incluyó el que mide la percepción sobre la situación actual del país, donde solo el 14\% de la población considera que es buena o muy buena. Ambos son factores cruciales en la valoración que dan los ciudadanos a la democracia y permiten explicar cuánto funciona o no el sistema político (Schumpeter, 1942; Sen, 1999), incluso el mismo informe del Latinobarómetro señala:

El problema más importante del país son los problemas de carácter económico, que son los que más se señalan en la región, por encima de la inseguridad y la delincuencia. En $2017,23 \%$ por ciento de los latinoamericanos consultados afirman que el principal problema del país es de naturaleza económica (salarios, empleo, inflación...), mientras que otro $11 \%$ adicional señala a 
la economía en general o la situación financiera. En total, 34\%, uno de cada tres latinoamericanos, afirma que el principal problema que enfrenta su país es de tipo económico. (Latinobarómetro, 2017, p. 58)

La seguridad económica, expresado en un correcto funcionamiento de la economía, unido a la percepción de estabilidad y desarrollo de un país por parte de sus ciudadanos, constituye un elemento clave, que permitirá la confianza ciudadana en sus gobernantes, y la labor de aquellas instituciones que lo representan. Esto nos indica la estrecha relación entre la estructura económica de un sistema y la expresión política que lo respalda. Solo en la medida que los ciudadanos perciban la representación política y no la representación de unos pocos, podrán experimentar satisfacción con la democracia como sistema de gobierno.

Sin embargo, para Ranciere (2012, p. 61): "Los males de los que sufren nuestras 'democracias' son, antes que nada, los males ligados al apetito insaciable de los oligarcas. (...)". Razón por la que plantea que no vivimos en democracia y hasta el momento esta nunca ha existido, sino que "vivimos en Estado de derecho oligárquicos, es decir, en Estados donde el poder de la oligarquía está limitado por el doble reconocimiento de la soberanía popular y de las libertades individuales". Si bien, no se sostiene la tesis que nunca ha existido la democracia, sino más bien que los sistemas democráticos han poseído y poseen un limitado carácter, respecto a su expresión teórica o significación en la realidad práctica de su manifestación en los distintos escenarios históricos. La democracia bajo el principio liberal se ha enarbolado como un sistema ideal para toda sociedad, sin embargo, sus expresiones concretas se apartan de ese ideal y expresan lo que se reconoce como la insatisfacción con el funcionamiento de la democracia como sistema de gobierno.

\section{Conclusiones}

La satisfacción con la democracia es un reflejo de las acciones y políticas que los gobiernos entablan, las cuales pueden ser valoradas positiva o negativamente, por esa razón, nuestros resultados comprueban la hipótesis que tanto los factores de rendimiento económico, como los políticos, así como la percepción de corrupción explican la satisfacción con el funcionamiento de la democracia en Latinoamérica. De ahí que podamos señalar que 
la baja percepción en estos factores debido a su posible mal funcionamiento, están disminuyendo esta percepción.

Un elemento a destacar, fue que, la percepción de la desigualdad en los ingresos, considera que se gobierna para unos cuantos grupos poderosos, la corrupción, está correlacionada con la baja confianza en instituciones del gobierno, lo cual indica una posible área de nuevos estudios. Los hallazgos en el modelo, permite comprobar con la literatura revisada el valor de su utilización en estudios sobre el funcionamiento de democracia. Específicamente en Latinoamérica, donde hoy pueden verse los fraccionamientos democráticos al no erradicar problemas sociales como la desigualdad, aunque se hayan disminuido los índices de pobreza extrema como en los casos de Bolivia, Chile, o Ecuador. Sin duda, estos problemas han influido en el descenso consecutivo de la satisfacción con el funcionamiento de la democracia. A pesar de ello, la mayoría de la población sigue considerando que la democracia a pesar de los problemas que ésta tiene, es la mejor forma de gobierno. Los desafíos están en su correcta implementación, cuando ésta deje de responder al interés económico de unas minorías como lo muestran los datos en Latinobarómetro y, en cambio, pase a dignificar al ciudadano, ese que es dador del poder democrático.

Lo anterior se explica por el sistema de gobierno que ha imperado bajo una forma inicialmente liberal y hoy en día bajo la hegemonía neoliberal, que ha mercantilizado los derechos ciudadanos y con ellos la democracia real como ejercicio de gobierno del llamado pueblo. Sin embargo, es de reconocer que han existido procesos de democratización, independientemente de los períodos de regímenes autoritarios en la región. El hecho de haber transitado por regímenes de bienestar en diferentes períodos ha contribuido a elevar los niveles de calidad de vida y con ello la aceptación de la democracia, a pesar de que estos regímenes, no escapan del funcionamiento sistémico capitalista, por tanto, a los reajustes del capital por consolidarse.

Finalmente, es importante señalar que el rol de las instituciones es fundamental en la percepción ciudadana, pero hay que añadir que, al tratarse de percepción, también influye en ello la visión que los medios de comunicación hacen ver y muestran sobre dichas instituciones, influyendo en cierta medida en la valoración que se tendrá sobre ellas. En este caso, ni los medios ni la percepción ciudadana escapan de un posicionamiento ideológico, que excede el espacio individual y subjetivo, pues están sujetos al funcionamiento de las hegemonías. 


\section{Bibliografía}

Aarts, A. y J. Thomassen (2008). Satisfaction with democracy: Do institutions matter? Electoral Studies, 5-18.

Anderson, C., \& Guillory, C. (1997). Political Institutions and Satisfaction with Democracy: A Cross-National Analysis of Consensus and Majoritarian Systems. The American Political Science Review, 91(1).

Ayala, J. (2002). Instituciones y economía una introducción al neoinstitucionalismo económico. México: Fondo de Cultura Economica.

Barreda, M. (2011). La calidad de la democracia: Un análisis comparado de América Latina. Política y gobierno, 265-295.

Canache, D., Mondak, J., \& Sligson, M. (2001). Meaning and Measurement in Cross-National Research on Satisfaction With Democracy. American for Public Opinion research.

Carlin, R. (2006). The Socioeconomic Roots of Suport for Democracy and the Quality of Democracy in Latin America. Revista de Ciencia Política, 26(1).

Cea, C., Alister, C., \& Guerrero, A. (2015). Democracia en Latinoamérica ¿qué factores influyen en la satisfacción y apoyo a la democracia? Fronteras, II(1), 85-113.

Cereceda-Marambio, K., \& Torres-Solís, A. (2017). Satisfacción con la democracia en Chile: De lo normativo a lo valorativo. Revista de Sociología, 32-49.

Dávalos, P. (2008). Neoliberalismo político y ‘Estado social de derecho'. Rebelión. Recuperado de: https://bit.ly/2OT18py (2019-08-20).

Díaz, A. (2014). La (in)satisfacción con el funcionamiento de la democracia en América Latina. Iberoamericana, 54, 169-173.

Dunn, J. (2014). Libertad para el pueblo. Historia de la democracia. Ciudad de México: FCE.

Espino, J. (2002). Fundamentos institucionales del mercado. Ciudad de México: UNAM.

Galli, C. (2013). El malestar de la democracia. Buenos Aires: FCE.

Latinobarómetro (2017). Latinobarómetro. (18 de octubre de 2018).

Lipset, S.(1959). Some Social Requisites of Democracy: Economic Development and. The American Political Science Review.

Morales, M. (2009). Corrupción y democracia: América Latina en perspectiva comparada. Gestión y política pública, 205-252.

O’Donnell, G. (2001). Accountability horizontal. La institucionalización legal de la desconfianza política. Revista de teoría y filosofía del derecho, 14, 7-31. 
Ranciere, J. (2012). El odio a la democracia. Buenos Aires: Amorrortu.

Scott, M., \& Pérez-Liñán, A. (2008). Regime Legacies and democratization explaining. Working Paper de Kellog Institute for International Studies.

Schumpeter, J. (1942). Capitalismo, socialismo y democracia. Ciudad de México: Folio.

Sen, A. (1999). Desarrollo y libertad. Buenos Aires: Planeta.

Sotillo, I. (2015). Instituciones y satisfacción con la democracia. Un estudio comparado de los vínculos representativos en Latinoamérica. Latinoamericana de Política comparada CELAEP, 129-157

Zovatto, D. (2011). Democracia y gobernabilidad en américa Latina. En el siglo XXI temprano. México: Tribunal Electoral del Poder Judicial de la Federación.

Zovatto, D. (2018). El estado de las democracias en América Latina a casi cuatro décadas del inicio de la Tercera Ola Democrática. Revista Derecho electoral, 25.

Fecha de envío: 2019/10/3I; Fecha de aceptación: 2020/02/10;

Fecha de publicación: 2020/03/01 